\title{
"Working Alliance Inventory" Versión Observacional: Traducción, Adaptación y Validación al Castellano
}

\author{
"Working Alliance Inventory" Observational form: Translation, Adaptation and \\ Validation to Spanish
}

\author{
Carlos Vöhringer C. \\ Pontificia Universidad Católica de Chile, Chile \\ J. Carola Pérez \\ Universidad del Desarrollo, Chile \\ Claudio Martínez \\ Universidad Diego Portales. \\ Carolina Altimir \\ Paula Dagnino \\ Nicolás Suárez \\ Mariane Krause \\ Pontificia Universidad Católica de Chile, Chile
}

(Rec: 12 de marzo de 2012 / Acept:12 de abril de 2013)

\begin{abstract}
Resumen
La investigación ha demostrado la fuerte relación entre alianza terapéutica y resultados en psicoterapia. Una buena alianza está asociada a mejores resultados en psicoterapia. Para medir alianza se han desarrollado una serie de instrumentos para terapeutas, pacientes, y observadores. Los instrumentos observacionales son menos intrusivos, disminuyendo los posibles efectos que las mediciones tendrían en el tratamiento. Además, han resultado más válido en algunas poblaciones clínicas. En Chile no se cuenta con un instrumento confiable y validado que mida alianza terapéutica desde la perspectiva de un observador. El presente artículo da cuenta de la traducción al castellano, y la adaptación y validación del Work Alliance Inventory (WAI-0) en su versión observacional para Chile. El (WAI-0) es un instrumento válido y confiable para medir alianza terapéutica. Palabras Clave: Alianza Terapéutica, Adaptación, Validación, Instrumento Observacional.
\end{abstract}

\begin{abstract}
Research has shown the strong association between therapeutic alliance and therapeutic outcomes. A good alliance is linked with better outcomes in psychotherapy. A number of instruments have been developed to measure the alliance aimed at therapists, patients, and observers. Observational instruments are less intrusive, and reduce the possible effects that measurements may have on treatment. In addition, they have proven to be more valid in some clinical populations. In Chile, there are no reliable and validated instruments to measure the therapeutic alliance from the point of view of an observer. The present article introduces a translation into Spanish along with an adaptation and validation for Chile of the Work Alliance Inventory (WAI-O) in its observational version. WAI-O is a valid and reliable instrument for measuring the therapeutic alliance. Key words: Working Alliance Inventory, Adaptation, Validation, Observational Form.
\end{abstract}

Correspondencia: Escuela de Psicología, Pontificia Universidad Católica de Chile. Vicuña Mackenna 4860, Macul. Santiago, Email de contacto: cmvohrin@uc.cl

El estudio se desarrolló en el marco del Proyecto FONDECYT 1080136, titulado "Interacción terapéutica, expresión de emociones y alianza terapéutica: Estudio de los ingredientes esenciales para el cambio en psicoterapia". 


\section{Introducción}

Un gran número de investigaciones realizadas en los últimos 25 años han dado cuenta de manera consistente de la fuerte relación entre alianza terapéutica y resultados en psicoterapia (Horvath y Symonds, 1991). Una elevada alianza terapéutica entre paciente y terapeuta está asociada a mejores resultados en el tratamiento de variados problemas de salud mental, como la depresión (Castonguay et al., 1996), desórdenes de personalidad (Hellerstein et al., 1998), y dependencia al alcohol (Conners et al., 1997). Estudios sobre la eficacia en psicoterapia y los factores asociados a los resultados terapéuticos, han concluido que la relación terapéutica explica un $30 \%$ de la mejoría de los pacientes (Lambert y Barley, 2002), y que la alianza terapéutica explica entre un $25 \%$ y un $30 \%$ de la varianza de los resultados de la terapia (Horvath, Del Re, Flückiger, y Symonds, 2011).

Para medir la alianza terapéutica se han desarrollado una serie de instrumentos en modalidad de autoreporte tanto para el terapeuta como para el paciente, así como para observadores externos. En la mayoría de los estudios, el autoreporte del paciente ha demostrado ser la modalidad más predictiva de los resultados del tratamiento, seguido por el reporte de un observador externo y finalmente el reporte del terapeuta (Horvath y Symonds, 1991). Sin embargo, entre algunas poblaciones clínicas, las puntuaciones de un observador pueden ser más válidas. Por ejemplo, en población adolescente (Shelef y Diamond, 2008), y en adultos que abusan de sustancias (Fenton, Cecero, Nich, Frankforter, y Carroll, 2001), la mejor elección para medir alianza son los instrumentos completados por observadores. Además, este tipo de instrumentos puede ser preferido por investigadores que estudian los cambios dentro de la sesión de terapia, en particular aquéllos que requieren de múltiples medidas de la alianza en el curso de una sesión de psicoterapia, y que por ende corren el riesgo de interrumpir el proceso terapéutico. De hecho, usar mediciones de la alianza desde la perspectiva de un observador es menos intrusivo y disminuye la amenaza de que la medición pueda tener efectos en el tratamiento.

En Chile no se cuenta con un instrumento confiable y validado que mida alianza terapéutica desde un observador externo al proceso de psicoterapia. Ante la ausencia de instrumentos nacionales que permiten cuantificar el constructo de alianza terapéutica desde la perspectiva de un observador, el presente estudio tiene por objetivo traducir al español, adaptar y validar el Working Alliance Inventory, forma observacional (Inventario de Alianza Terapéutica, WAI-O) desarrollado por Horvath y Greenberg (1986). Este inventario mide la alianza terapéutica desde la perspectiva de un observador y cuenta con amplio respaldo por sus condiciones psicométricas en estudios internacionales. Asimismo, las versiones de autoreporte de paciente y terapeuta de este instrumento cuentan con estudios de validez en nuestro país (Santibáñez, 2001), lo que facilitaría obtener la validez concurrente del WAI-O.

\section{Instrumentos de Medición de Alianza Terapéutica}

Aunque la relación entre la alianza y resultado del proceso terapéutico está bien establecida, hay poco acuerdo sobre cuál es la mejor manera de medir alianza terapéutica. Horvath y colaboradores encontraron más de 30 medidas de alianza, sin contar entre ellas las diferentes versiones de los mismos instrumentos (Horvath et al., 2011). Actualmente existen al menos 11 instrumentos de mayor uso, los cuales varían en perspectiva (observador, cliente, o terapeuta) y orientación teórica (Fenton et al., 2001).

Entre los instrumentos más utilizados y con validación empírica se encuentran:

1. California Psychotherapy Alliance Scales (CALPAS; Hartley y Strupp, 1983). Este instrumento está basado en una perspectiva psicodinámica de la alianza y en los trabajos de Bordin (1979). Se compone de 24 ítems agrupados en cuatro sub-escalas: a) Capacidad de trabajo del paciente, b) Compromiso del paciente, d) Acuerdo entre paciente y terapeuta sobre los objetivos de la terapia y las estrategias para alcanzarlos, y d) Comprensión e involucramiento del terapeuta con el proceso terapéutico. La consistencia interna reportada por los autores en la versión paciente es de 0.73 y la versión terapeuta es de 0.95 .

2. Penn Helping Alliance Questionnaire (HAq; Alexander y Luborsky, 1986). Este instrumento da cuenta de dos conceptualizaciones de alianza. El tipo I refleja la mirada psicoanalítica del vínculo, centrada en el vínculo del paciente con el terapeuta. El tipo II está relacionado con el concepto de alianza de Bordin (1979), refiriéndose a la idea de un mutuo acuerdo entre terapeuta y paciente sobre las metas y las tareas de la terapia. Está compuesto por 19 ítems y cuenta con una versión para terapeutas y otras para pacientes. La consistencia interna del instrumento es de 0.90 y 0.93 para cada una de las versiones (Barber et al., 1999).

3. Vanderbilt Therapeutic Alliance Scale (VTAS; Hartley y Strupp, 1983). Este instrumento representa una combinación de marcos teóricos psicodinámicos y eclécticos. Su modelo considera que una alianza exitosa se basa en la presencia o ausencia de los siguientes 5 factores: clima positivo, intrusividad del terapeuta, resistencia o ansiedad del paciente, motivación del paciente y responsabilidad del paciente. El instrumento está compuesto de 44 ítems, que se 
distribuyen en tres sub-escalas: a) Contribución del terapeuta a la alianza, b) Contribución del paciente a la alianza, c) interacción paciente-terapeuta. Los autores reportan una consistencia interna de 0.93 .

4. Working Alliance Inventory (WAI).Dentro de las escalas más ampliamente utilizada para medir la alianza terapéutica se encuentra el Working Alliance Inventory (WAI) desarrollado por Horvath y Greenberg (1986). Este instrumento se elaboró basado en la conceptualización de alianza de Bordin (Hatcher y Gillaspy, 2006). Bordin (1979) planteó que una buena alianza es un prerrequisito para el cambio en todas las formas de psicoterapia. El aspecto central de la teoría de Bordin es concebir a la alianza como el calce de la colaboración entre el cliente y el terapeuta, e identificando tres componentes que la configuran: (a) acuerdo entre paciente y terapeuta en las tareas, (b) calidad del vínculo entre paciente y terapeuta y, (c) acuerdo en los objetivos de la terapia. Las tareas se refieren a las acciones y pensamientos que forman parte del trabajo en el proceso terapéutico, de modo que la percepción de estas acciones o tareas como relevantes para la mejoría es una parte importante del establecimiento de la alianza. Además, la existencia de acuerdo entre terapeuta y paciente respecto a cuáles son los objetivos a alcanzar con la psicoterapia, así como compartir mutuamente confianza y aceptación, son elementos esenciales para una buena alianza (Corbella y Botella, 2003).

El cuestionario posee tres versiones: una para terapeutas (WAI-T), otra para pacientes (WAI-P) y una forma observacional (WAI-O). El cuestionario consta de 36 ítems divididos en tres subescalas: vínculo, tareas y metas. Cada escala consta de 12 ítems, puntuados en una escala tipo Likert de 7 puntos $(1=$ Nunca hasta $7=$ Siempre $)$. Los inventarios para paciente y terapeuta han sido traducidos, adaptados y validados en Chile por Santibañez (2001). La confiabilidad de la versión del terapeuta es de $0.67,0.80$, 0.78 y 0.93 (Cronbach alfa) para las sub-escalas de vínculo, tareas, metas y el total (respectivamente). En la versión del paciente la confiabilidad alcanza los coeficientes de 0.70 , $0.85,0.88$ y 0.90 para las sub-escalas de vínculo, tareas, metas y el total respectivamente.

\section{Working Alliance Inventory (WAI) - Versión Observacional}

El Working Alliance Inventory, en su versión para observadores (WAI-O), desarrollado por Horvath (1994), se utiliza para medir el grado de alianza terapéutica entre el terapeuta y el paciente dentro de la sesión. Es un instrumento que puede ser administrado fácilmente y con rapidez por un observador no participante. Sus resultados han demostrado compartir un porcentaje significativo de varianza con otros instrumentos que miden alianza terapéutica (Hanson et al., 2002). El WAI-O ha demostrado ser confiable y válido para medir la alianza terapéutica (Horvath y Greenberg, 1986). Por ejemplo, el WAI-O ha mostrado una consistencia interna de 0.98 y una confiabilidad inter jueces de 0.92 (Fenton et al, 2001; Tichenor y Hill, 1989).

\section{Método}

\section{Participantes}

En el presente estudio se utilizó una muestra de 60 sesiones de psicoterapia, desarrolladas por 15 diadas paciente-terapeuta. Las diadas se seleccionaron por conveniencia desde diferentes centros de atención psicológica de la ciudad de Santiago de Chile. La díada para formar parte del estudio debía estar conformada por un paciente mayor de edad, y encontrarse al menos en la tercera sesión de psicoterapia.

En relación a las características de las diadas, el 21\% de ella estaba conformada por parejas donde paciente y terapeutas eran mujeres, y en el $79 \%$ restantes el sexo de paciente y terapeuta estaba combinado. No se presentaron diadas donde ambos (paciente y terapeuta) fueran de sexo masculino. El promedio de edad de los pacientes fue de 36.40 años (DS $=11.65$ años), con un rango de 20 a 65 años. Se distribuyen homogéneamente por sexo (53.3\% Mujeres). Los terapeutas presentan en promedio 11.47 años de experiencia ( $\mathrm{DS}=6.38$ años), con un rango de 3 a 20 años de experiencia. Un $73.33 \%$ de los terapeutas es de sexo femenino y un $26.66 \%$ masculino (ver Tabla 1 ).

Respecto al motivo por el que consultan, un $53.33 \%$ de los pacientes lo hace por problemas asociados al consumo de alcohol u otra droga, un $20 \%$ por sintomatología ansiosa depresiva y un $13.33 \%$ por intento de suicidio. Además se presenta un caso por problema de pareja y un caso por violencia en la familia.

Se incluyeron en el estudio 60 sesiones videadas de las 14 diadas,. Respecto al número de la sesión -dentro del proceso terapéutico- en que fueron aplicados los instrumentos, la media correspondió a la sesión 22 (DS= 20.80). Un $30 \%$ de las sesiones utilizadas en la muestra se encuentran entre la sesión 3 a la 9 de un proceso psicoterapéutico, un $31.6 \%$ entre las sesiones 10 y 19 , un $21 \%$ entre las sesiones 20 y 39 , y un $16.7 \%$ sobre la sesión 40 de un proceso de psicoterapia. 
Tabla 1. Características de la muestra.

\begin{tabular}{lcccccc}
\hline Terapia & \multicolumn{5}{c}{ Terapeuta } & \multicolumn{2}{c}{ Paciente } \\
\hline Terapia & Sesiones $^{\mathrm{a}}$ & Sexo & Años Experiencia & Sexo & Edad & Motivo consulta \\
\hline 1 & 1 & $\mathrm{M}$ & 10 & $\mathrm{~F}$ & 40 & Abuso de drogas \\
2 & 1 & $\mathrm{~F}$ & 8 & $\mathrm{~F}$ & 38 & Abuso de drogas \\
3 & 4 & $\mathrm{M}$ & 20 & $\mathrm{~F}$ & 37 & Intento suicidio \\
4 & 1 & $\mathrm{~F}$ & 4 & $\mathrm{M}$ & 27 & Abuso de drogas \\
5 & 1 & $\mathrm{~F}$ & 8 & $\mathrm{M}$ & 27 & Abuso de drogas \\
6 & 1 & $\mathrm{~F}$ & 8 & $\mathrm{M}$ & 41 & Abuso de drogas \\
7 & 1 & $\mathrm{~F}$ & 4 & $\mathrm{M}$ & 43 & Abuso de drogas \\
8 & 5 & $\mathrm{~F}$ & 15 & $\mathrm{M}$ & 27 & Problema de pareja \\
9 & 4 & $\mathrm{M}$ & 3 & $\mathrm{M}$ & 20 & Violencia familiar \\
10 & 10 & $\mathrm{M}$ & 20 & $\mathrm{~F}$ & 32 & Sint. Ansiosa-depresiva \\
11 & 6 & $\mathrm{~F}$ & 20 & $\mathrm{~F}$ & 65 & Intento suicidio \\
12 & 1 & $\mathrm{~F}$ & 8 & $\mathrm{M}$ & 27 & Abuso de drogas \\
13 & 1 & $\mathrm{~F}$ & 8 & $\mathrm{~F}$ & 51 & Abuso de drogas \\
14 & 19 & $\mathrm{~F}$ & 20 & $\mathrm{~F}$ & 27 & Sint. Ansiosa-depresiva \\
15 & 4 & $\mathrm{~F}$ & 16 & $\mathrm{~F}$ & 44 & Sint. Ansiosa-depresiva \\
\hline
\end{tabular}

Nota. ${ }^{\text {a }}$ Número de sesiones de la terapia (díada) incluidas en el análisis. M= Masculino, F= Femenino.

\section{Instrumentos}

Working Alliance Inventory (WAI-O). Se tradujo y adaptó el Working Alliance Inventory (WAI; Horvath y Greenberg, 1986) en su versión para observadores (WAI-O). Este instrumento fue originalmente desarrollado como una adaptación de las versiones de paciente y terapeuta, las que fueron redactadas en primera persona singular y traspasadas a la tercera forma del plural en su forma observacional (Horvath, y Greenberg, 1986). Como fue mencionado anteriormente, el cuestionario consta de 36 ítems divididos en tres sub-escalas: vínculo, tareas y metas. Cada escala consta de 12 ítemes, puntuadas en una escala tipo Likert de 7 puntos $(1=$ Nunca hasta $7=$ Siempre $)$.

Inventario de Alianza de Trabajo (WAI; Horvath y Greenberg, 1986). Incluye versiones para terapeutas (WAI-T) y pacientes (WAI-P). Ambos cuestionarios constan de 36 ítems divididos en tres subescalas de 12 ítems cada una, puntuadas en una escala tipo Likert de 7 puntos (a mayor puntaje mayor alianza terapéutica). Las tres sub-escalas: metas, tareas y vínculo, están basadas en la teoría de la alianza terapéutica de Bordin (1979). Ambos cuestionarios han sido traducidos, adaptados y validados en Chile por Santibañez (2001), con coeficientes de confiabilidad para la versión del terapeuta de $0.67,0.80,0.78$ y 0.93 para las sub-escalas de vínculo, tareas, metas y total respectivamente, y para la versión del paciente con coeficientes de $0.70,0.85$,
0.88 y 0.90 para las sub-escalas de vínculo, tareas, metas $\mathrm{y}$ total respectivamente.

Cuestionario para la evaluación de resultados y evolución en psicoterapia (OQ.45.2; Lambert, 1996). El cuestionario da cuenta de de los resultados y evolución en psicoterapia. Se compone de 45 ítems, dividido en tres sub-escalas: Sintomatología, Relaciones Interpersonales y Rol Social. La escala de sintomatología evalúa síntomas depresivos y angustiosos, además de buscar la detección de abuso de drogas y alcohol; la escala de relaciones interpersonales mide tanto la satisfacción como los problemas en las relaciones interpersonales; $y$ la escala de rol social mide conflicto e insatisfacción del paciente en tareas relacionadas con el empleo, roles familiares y ocio. Cada ítem se puntúa en una escala Likert de nivel ordinal de 0 a 4 puntos (rango del puntaje del OQ total va de 0 a 180 puntos). El puntaje del OQ.45.2 se obtiene sumando el puntaje obtenido en cada ítem, y entrega un puntaje total y uno para cada escala. Un mayor puntaje en la escala indica un mayor disconfort por parte del paciente.

El cuestionario fue traducido, adaptado y validado en Chile por Von Bergen y De la Parra (2002), con índices de confiabilidad de $0.89,0.67,0.63$ y 0.91 para las escalas de sintomatología, relaciones interpersonales, rol social y el total respectivamente

Vanderbilt Therapeutic Alliance Scale (VTAS-R, SF). En el presente estudio se utiliza la versión breve desarrollada por Shelef y Diamond (2008), que consta de 5 ítemes que miden 
alianza general, puntuados en una escala tipo Likert de 0 a 5 puntos. La forma abreviada está altamente correlacionada con la forma original (de 26 ítems) y presentó una confiabilidad de 0.87 . Este cuestionario no ha sido traducido ni validado en Chile.

\section{Diseño}

Se empleó un diseño de tipo correlacional. Con el fin de establecer la validez concurrente y divergente del instrumento WAI-O, se determinó su relación con otros instrumentos que miden el mismo constructo (WAI-P, WAI-T y VTAS-R SF) y constructos diferentes (OQ-45.2, que mide resultados). Además, se incluyeron instrumentos que miden alianza terapéutica utilizando dos estrategias de medición diferentes (autoreporte v/s observacional). Dado que el instrumento WAI-O mide alianza terapéutica, se espera una relación positiva y significativa con los instrumentos que miden el mismo constructo (Validez Concurrente). Adicionalmente, se espera que este instrumento presente la mayor correlación con el instrumento VTAS, el cual -al igual que el WAI-O- mide la alianza terapéutica desde la perspectiva de un observador. Finalmente, se espera que la alianza se relacione con los resultados terapéuticos (medidos a través del OQ.45.2), pero conmenor magnitud que la que se establece entre los instrumentos que miden el constructo alianza (Validez Discriminante).

\section{Procedimiento}

El estudio consideró las siguientes etapas: a) Traducción del instrumento, y determinación de su validez de contenido, b) Pilotaje del instrumento, c) Determinación de propiedades psicométricas de la escala: Validez y Confiabilidad.

De la traducción. Un traductor profesional tradujo el WAI-O desde su versión original en inglés al español, generándose la primera versión del WAI-O. Se formó un comité de expertos para determinar la validez de contenido del instrumento, compuesto por cinco psicoterapeutas bilingües. A los expertos se les entregó la versión original del WAI-O y su traducción al español realizada por el traductor. Se les solicitó su apreciación acerca de la adecuación de la traducción y sugerencias para mejorar la redacción de los ítemes en el marco conceptual de la alianza terapéutica. Es importante indicar que los jueces señalaron más de una subescala al atribuir a qué dimensión de alianza correspondía cada ítem. Las observaciones entregadas por los expertos fueron consolidadas por el equipo de investigación, generándose una versión final traducida, la cual fue re-traducida al inglés (método re-traducción). Una vez analizada la re-traducción se conformó la segunda versión del instrumento que fue utilizada en la etapa de pilotaje.

Del Pilotaje. Posteriormente se realizó la aplicación piloto del WAI-0 (versión 2) a 10 sesiones de psicoterapia grabadas en video, por dos duplas de psicoterapeutas entrenados en su aplicación. Esta etapa fue desarrollada para determinar y corregir las dificultades que podrían presentarse en la aplicación del instrumento por parte de terapeutas observadores, y para establecer una confiabilidad adecuada entre los evaluadores (quienes completaron el instrumento en la estudio de validez del instrumento). Inicialmente se codificaron tres sesiones en conjunto con los cuatro evaluadores, se discutieron los criterios utilizados por ellos y se obtuvo la confiabilidad entre sus puntajes. Las siguientes evaluaciones se hicieron de forma independiente y se revisaron en conjunto cada tres sesiones. En esta etapa se modificó la opción de respuesta del instrumento de una escala Likert de 7 puntos a una de 5 puntos, eliminándose las opciones de respuesta Casi Siempre y Casi Nunca, dada la dificultad de los observadores para distinguirlas de las categorías adyacentes. Al final de esta etapa se elaboró el instrumento "Working Alliance Inventory" en su versión en español para observadores (WAI-O, versión final).

Un aspecto a resaltar de esta etapa fue la dificultad que reportaron los jueces para poder responder algunos ítemes del WAI-O al observar una sesión videograbada. Los jueces señalaron que les resultó más fácil responder preguntas relacionadas con la sub-escala vínculo, que aquellas correspondientes a las escalas de metas y tareas.

Determinación de Propiedades psicométricas de la escala: Validez y Confiabilidad. Para la aplicación de los instrumentos a la muestra de sesiones de psicoterapia, primero se tomó contacto con terapeutas de centros de atención psicológica. Se les informó el sentido de la investigación y se le solicitó su colaboración en el estudio. Los pacientes invitados a participar fueron seleccionados entre los pacientes que estos terapeutas estaban atendiendo en sus centros y que llevaban al menos tres sesiones de terapia. Los terapeutas que aceptaron participar solicitaron el consentimiento a sus respectivos pacientes y les explicaron el sentido de la investigación. Una vez que los pacientes aceptaban participar en el estudio, el terapeuta desarrolló el proceso de consentimiento informado. Para ello el terapeuta solicitó a sus pacientes leer y firmar el documento de Consentimiento Informado, que presentaba información sobre el objetivo del estudio y las actividades que involucraba para los pacientes, específicamente la videograbación de las sesiones y los cuestionarios que debía contestar. 
Respecto a otros aspectos éticos del estudio, la participación en el mismo fue voluntaria (tanto para los terapeutas como los pacientes), se aseguró la confidencialidad de los datos personales de paciente y terapeuta, además del resguardo de la sesión videada. Además, se aseguró al paciente que la información entregada sobre la evaluación de la alianza no sería entregada a su terapeuta.

$\mathrm{Al}$ momento de la recolección de los datos, el terapeuta completaba una hoja de datos con identificación del paciente (sexo, edad, motivo de consulta), del terapeuta (orientación clínica, sexo y años de experiencia) e identificación del número de sesión dentro del proceso terapéutico que se video-grababa. Adicionalmente a la grabación en video de la sesión, el terapeuta solicitaba al paciente que contestara los instrumentos OQ.45.2 y WAI-P. Al igual que el paciente, una vez concluida la sesión, el terapeuta también completaba el WAI-P.

Posteriormente, dos jueces observaron la grabación en video de cada sesión y completaron el WAI-O para esa sesión. Las puntuaciones de ambos observadores fueron promediadas para obtener los puntajes finales. Adicionalmente, un observador independiente observó cada sesión videograbada y le administró el VTAS-SF.

Los datos de los instrumentos aplicados a cada sesión de psicoterapia fueron analizados utilizando el software SPSS.v18. Se realizaron análisis descriptivos de los datos, estimándose el Promedio y DS de cada una de las escala. Para el análisis de la validez concurrente y divergente de los instrumentos se estimaron correlaciones parciales entre las variables, controlándose el avance del proceso terapéutico al incorporar como co-variable el número de la sesión del proceso terapéutico. Para determinar las diferencias entre las correlaciones obtenidas se utilizó la prueba de diferencia de correlaciones utilizando la transformación a valores $\mathrm{Z}$ de Fisher. Finalmente, para la estimación de la confiabilidad de la escala WAI-O se utilizó el método de consistencia interna (alfa de Cronbach).

\section{Resultados}

\section{Resultados Descriptivos}

Los resultados descriptivos se presentan en la Tabla 2. Destaca que los pacientes y terapeutas de la muestra reportan en promedio un nivel alto de alianza terapéutica en las sesiones, tanto en términos de la alianza global como por sub-escalas. También destacan los altos puntajes promedios de la alianza terapéutica medida desde la modalidad de un observador, tanto a nivel global como por sub-escalas, lo que concuerda con las percepciones de terapeutas y pacientes respecto a la alianza. Lo mismo sucede al medir la alianza con para el instrumento VTAS-SF.

Validez Concurrente y Divergente de la Escala WAI-O: Escalas Totales. En concordancia con lo esperado teóricamente, se presentan correlaciones positivas, estadísticamente significativas y de magnitud moderada entre los instrumentos que miden el constructo de Alianza Terapéutica, al ser reportada por pacientes, terapeutas y observadores. De hecho, la escala WAI-O presenta correlaciones significativas con las versiones que miden alianza desde la perspectiva del observador (VTAS), del paciente y terapeuta. La única excepción lo constituye el VTAS-SF, el cual solo se relaciona en forma significativa con la alianza percibida por observadores $(r=$ $.42, p=.002)$, en cambio, las correlaciones entre escala y la alianza reportada por pacientes y terapeutas no alcanzan significación estadística.

La escala WAI-O no se relacionó en forma estadísticamente significativa con los resultados terapéuticos. Similar patrón se presentó al considerar la alianza terapéutica medida por observadores a través del VTAS. En cambio, la alianza medida a través del auto-reporte de pacientes y/o terapeutas sí se relaciona en forma significativa con los resultados terapéuticos. Así, a mayor alianza reportada por estos actores del proceso terapéutico menor es la sintomatología reportada por el paciente.

$\mathrm{Al}$ analizar según el método de recolección de datos y considerando los instrumentos que miden Alianza Terapéutica, se aprecia que las versiones de autoreporte (WAI paciente y terapeuta) presentan una correlación de magnitud grande $(r=$ $.86, p \leq .001$ ) entre sí. Esta correlación es de mayor magnitud que aquella encontrada al considerar la alianza medida a través de diferentes métodos de recolección (observacional v/s autoreporte). Por ejemplo, la alianza medida a través del autoreporte del paciente se relaciona con la medición observacional de la alianza (WAI-O) en una magnitud moderada $(r=.45)$, correlación de inferior magnitud que la antes mencionada $(z=3.99, p \leq .001)$. Similar resultado se presenta al compararla con la relación encontrada entre la alianza reportada por el terapeuta y la medición observacional a través del WAI-O $(\mathrm{z}=4.16, p \leq .001)$.

Asimismo, al considerar el método de recolección observacional, la relación entre la alianza medida a través del VTAS-SF y el instrumento WAI-O $(r=.42)$ es de menor magnitud que la correlación encontrada entre las mediciones de alianza entre paciente y terapeuta, ambas recolectadas a través del autoreporte $(\mathrm{z}=4.20, p \leq .001)$.

Validez Concurrente de la Escala WAI-O: Sub-escalas. Las diferentes dimensiones de la alianza terapéutica medida desde el punto de vista del observador, utilizando 
Tabla 2. Datos descriptivos de las Variables: Alianza y Resultados Terapéuticos

\begin{tabular}{|c|c|c|c|c|c|}
\hline & $\mathrm{N}$ & Mínimo & Máximo & M & DS \\
\hline \multicolumn{6}{|l|}{ Resultados terapéuticos } \\
\hline Síntomas & 4 & 83 & 37.41 & 19.13 & 4 \\
\hline Relaciones. Interpersonales & 2 & 41 & 15.51 & 7.10 & 2 \\
\hline Rol Social & 2 & 25 & 11.61 & 5.09 & 2 \\
\hline Escala Total & 6 & 138 & 62.76 & 28.92 & 6 \\
\hline \multicolumn{6}{|c|}{ Alianza Terapéutica: Medición Observacional } \\
\hline WAI-O Vínculo & 60 & 28.50 & 60.00 & 54.61 & 5.26 \\
\hline WAI-O Tareas & 60 & 35.00 & 60.00 & 53.64 & 5.90 \\
\hline WAI-O Metas & 60 & 37.91 & 60.00 & 51.86 & 5.94 \\
\hline WAI-O Escala Total & 60 & 101.41 & 179.50 & 160.11 & 15.70 \\
\hline VTAS total & 59 & 12.00 & 25.00 & 19.90 & 3.22 \\
\hline \multicolumn{6}{|c|}{ Alianza Terapéutica: Medición Autoreporte } \\
\hline \multicolumn{6}{|c|}{ Paciente } \\
\hline WAI-P Vínculo & 58 & 49.00 & 84.00 & 72.46 & 9.31 \\
\hline WAI-P Tareas & 58 & 39.27 & 84.00 & 68.91 & 10.94 \\
\hline WAI-P Metas & 58 & 28.00 & 82.00 & 66.11 & 10.80 \\
\hline WAI-P Escala Total & 58 & 127.00 & 245.00 & 207.47 & 28.73 \\
\hline \multicolumn{6}{|l|}{ Terapeuta } \\
\hline WAI-T Vínculo & 55 & 55.00 & 83.00 & 71.51 & 2. 6.63 \\
\hline WAI-T Tareas & 55 & 44.00 & 82.00 & 64.02 & 10.28 \\
\hline WAI-T Metas & 55 & 45.00 & 81.00 & 63.54 & 10.49 \\
\hline WAI-T Escala Total & 55 & 153.00 & 242.00 & 199.07 & 25.82 \\
\hline
\end{tabular}

Tabla 3. Correlaciones Parciales entre las mediciones de Alianza Terapéutica (según cliente, terapeuta y observador) y Resultados Terapéuticos

\begin{tabular}{|c|c|c|c|c|c|}
\hline & 1 & 2 & 3 & 4 & 5 \\
\hline \multirow[t]{2}{*}{$\begin{array}{l}\text { 1. Resultados Terapéuticos } \\
\text { (OQ.45.2 Escala Total) }\end{array}$} & 1.00 & -.10 & $-.53 * * *$ & $-.44 * * *$ & -.10 \\
\hline & 59 & 59 & 57 & 54 & 59 \\
\hline \multirow[t]{2}{*}{ 2. Alianza según VTAS-SF } & & 1.00 & $.26^{\mathrm{t}}$ & .17 & $.42 * *$ \\
\hline & & 59 & 57 & 54 & 59 \\
\hline \multirow[t]{2}{*}{ 3. WAI versión Paciente Escala Total } & & & 1.00 & $.86^{* * *}$ & $.45^{* * *}$ \\
\hline & & & 58 & 55 & 58 \\
\hline \multirow[t]{2}{*}{ 4. WAI versión Terapeuta Escala Total } & & & & 1.00 & $.41 * *$ \\
\hline & & & & 55 & 55 \\
\hline 5. WAI versión Observador Escala total & & & & & 1.00 \\
\hline
\end{tabular}

Nota. Correlaciones parciales en que se controla el número de sesión en que son aplicados los instrumentos. 51 gl.en cada correlación estimada. $* * * \mathrm{p} \leq .001 ; * * \mathrm{p} \leq .01 ; * \mathrm{p} \leq .05 ;{ }^{\mathrm{t}} \mathrm{p} \leq 10$ 
el instrumento WAI-O, se relaciona en forma positiva, moderada y estadísticamente significativa con la alianza medida por un observador a través del VTAS. Asimismo, la alianza terapéutica reportada por un observador a través del VTAS se relaciona en forma estadísticamente significativa solo con la dimensión vínculo cuando es reportada por el paciente, $\mathrm{y}$-conforme a lo antes reportado-no se relaciona con las diferentes dimensiones de la escala cuando la alianza reportada por los terapeutas.

Además, al observar el comportamiento de las distintas subescalas del WAI-O, se aprecia que cada una de éstas se relaciona en forma positiva y significativa con las diferentes sub-escalas de la alianza medida a través del instrumento WAI en las versiones paciente y terapeuta. Por ejemplo, la dimensión tareas de la medición observacional se relaciona con todas las dimensiones de la alianza reportada por pacientes y terapeutas. La única excepción la constituye la correlación entra la dimensión metas reportada por el observador y la dimensión tareas reportada por el paciente (Tabla 4).

En cada uno de los instrumentos que miden alianza terapéutica (WAI-O, WAI-P y WAI-T) destaca la elevada correlación que presentan las diferentes sub-escalas entre sí. En la medición observacional las correlaciones entre las sub-escalas oscilan entre .64 a .92; en el reporte del paciente oscilan entre .75 a .84 , y en el reporte del terapeuta alcanzan valores de .76 a .91 .

Validez Discriminante de la Escala WAI-O: Sub-escalas. Finalmente, en concordancia con la ausencia de relación estadísticamente significativa entre resultados terapéuticos (Escala Total) y la Alianza Terapéutica medida a través del WAI-O (Escala Total), la mayoría de las correlaciones entre las sub-escalas del WAI-O no se relacionan con la escala total y sub-escalas del instrumento que mide resultados terapéuticos (Tabla 5). La única excepción se presenta en la

Tabla 4. Coeficiente de correlación parcial entre las mediciones de Alianza Terapéutica (según cliente, terapeuta y observador) y VTAS-SF

\begin{tabular}{|c|c|c|c|c|c|c|c|c|c|c|}
\hline & 1 & 2 & 3 & 4 & 5 & 6 & 7 & 8 & 9 & 10 \\
\hline \multirow[t]{2}{*}{ 1. Alianza según VTAS-SF } & 1.00 & $.30^{*}$ & $.44 * * *$ & $.41 * *$ & $.34 *$ & .14 & $.25^{\mathrm{t}}$ & .20 & .16 & .13 \\
\hline & 59 & 59 & 59 & 57 & 57 & 57 & 54 & 54 & 54 & 59 \\
\hline \multirow[t]{2}{*}{ 2. WAI versión Observador - Vínculo } & & 1.00 & $.72 * * *$ & $.64 * * *$ & $.45^{* * *}$ & $.29 *$ & $.49 * * *$ & $.57 * * *$ & $.36^{* *}$ & $.39 * *$ \\
\hline & & 60 & 60 & 60 & 58 & 58 & 58 & 55 & 55 & 55 \\
\hline \multirow[t]{2}{*}{ 3. WAI versión Observador - Tareas } & & & 1.00 & $.92 * * *$ & $.43^{* * *}$ & $.31^{*}$ & $.45^{* * *}$ & $.36^{* *}$ & $.31 *$ & $.32 *$ \\
\hline & & & & 60 & 60 & 58 & 58 & 58 & 55 & 55 \\
\hline \multirow[t]{2}{*}{ 4. WAI versión Observador - Metas } & & & & 1.00 & $.38 * *$ & $.26^{\mathrm{t}}$ & $.43 * * *$ & $.31 *$ & $.31 *$ & $.32 *$ \\
\hline & & & & & 58 & 58 & 58 & 55 & 55 & 55 \\
\hline \multirow[t]{2}{*}{ 5. WAI versión Paciente - Vínculo } & & & & & 1.00 & $.77 * * *$ & $.75^{* * *}$ & $.77 * * *$ & $.77 * * *$ & $.77 * * *$ \\
\hline & & & & & 58 & 58 & 58 & 55 & 55 & 55 \\
\hline \multirow[t]{2}{*}{ 6. WAI versión Paciente - Tareas } & & & & & & 1.00 & $.84 * * *$ & $.63 * * *$ & $.81 * * *$ & $.82 * * *$ \\
\hline & & & & & & 58 & 58 & 55 & 55 & 55 \\
\hline \multirow[t]{2}{*}{ 7. WAI versión Paciente - Metas } & & & & & & & 1.00 & $.58 * * *$ & $.76^{* * *}$ & $.77 * * *$ \\
\hline & & & & & & & 58 & 55 & 55 & 55 \\
\hline \multirow[t]{2}{*}{ 8. WAI versión Terapeuta - Vínculo } & & & & & & & & 1.00 & $.76^{* * *}$ & $.76^{* * *}$ \\
\hline & & & & & & & & 55 & 55 & 55 \\
\hline \multirow[t]{2}{*}{ 9. WAI versión Terapeuta - Tareas } & & & & & & & & & 1.00 & $.91 * * *$ \\
\hline & & & & & & & & & 55 & 55 \\
\hline 10. WAI versión Terapeuta - Metas & & & & & & & & & & 1.00 \\
\hline
\end{tabular}

Nota. Correlaciones parciales en que se controla el número de sesión en que son aplicados los instrumentos. 51 gl. en cada correlación estimada. *** $\mathrm{p} \leq .001 ; * * \mathrm{p} \leq .01 ; * \mathrm{p} \leq .05 ;{ }^{\mathrm{t}} \mathrm{p} \leq 10$ 
relación inversa que se estableció entre la dimensión vínculo percibida por un observador y el reporte de funcionamiento social del paciente (Rol Social).

Confiabilidad. La confiabilidad de la escala total de la medición observacional de la alianza, medida a través del WAI-O, es de 0.97 (Alfa de Cronbach). Asimismo, la confiabilidad de cada una de las escalas es buena, obteniéndose un alfa de 0.93 en la escala Vínculo, 0.94 en la escala Tareas y 0.95 en la escala Metas. Finalmente, el análisis realizado indica que eliminar ítems, tanto al considerar la escala total como cada una de las escalas, no mejora el nivel de confiabilidad obtenido.

\section{Discusión}

Los resultados mostraron que el instrumento traducido y adaptado presenta una adecuada validez y confiabilidad para medir alianza terapéutica desde la perspectiva de un observador en díadas paciente-terapeuta chilenos. En concordancia con lo esperado teóricamente, se presentaron correlaciones positivas, significativas estadísticamente y de magnitud moderada entre los instrumentos que miden el constructo de Alianza Terapéutica, al ser reportada por clientes, terapeutas y observadores. Además, este instrumento, al no relacionarse en forma significativa con el instrumento OQ.45.2, no daría cuenta de los resultados terapéuticos. Respecto a la confiabilidad, el instrumento obtuvo una buena consistencia interna, que es similar a la reportada por los autores (Tichenor y Hill, 1989).

Llama la atención que al considerar las diferentes versiones del WAI, tanto en su versión observacional, como en las versiones de reporte del paciente y terapeuta, las diferentes sub-escalas presentan elevadas correlaciones entre sí. Esta elevada correlación es concordante con la dificultad que presentaron los jueces evaluadores del instrumento para asignar la dimensión que era medida por cada ítem. En este sentido se podría señalar que este instrumento da una buena medida de alianza terapéutica a nivel global, pero no lograría discriminar entre las distintas sub-escalas (vínculo, tareas y metas) que el modelo Bordin distingue. Este patrón de resultado es consistente con los estudios que cuestionan la

Tabla 5. Coeficiente de correlación Parcial entre las mediciones de Alianza Terapéutica medidas a través de un observador y resultados terapéuticos.

\begin{tabular}{|c|c|c|c|c|c|c|c|c|}
\hline & 1 & 2 & 3 & 4 & 5 & 6 & 7 & 8 \\
\hline \multirow[t]{2}{*}{ 1. Síntomas } & 1.00 & $.78 * * *$ & $.64 * * *$ & $.81 * * *$ & -.12 & -.08 & -.07 & -.10 \\
\hline & & 59 & 59 & 59 & 59 & 59 & 59 & 59 \\
\hline \multirow[t]{2}{*}{ 2. Relaciones Interpersonales } & & 1.00 & $.74 * * *$ & $.63^{* * *}$ & -.20 & -.13 & -.13 & -.17 \\
\hline & & & 59 & 59 & 59 & 59 & 59 & 59 \\
\hline \multirow[t]{2}{*}{ 3. Rol Social } & & & 1.00 & $.73 * * *$ & $-.26^{*}$ & -.18 & -.14 & -.21 \\
\hline & & & & 59 & 59 & 59 & 59 & 59 \\
\hline \multirow[t]{2}{*}{ 4. OQ Escala Total } & & & & 1.00 & -.11 & -.08 & -.06 & -.09 \\
\hline & & & & & 59 & 59 & 59 & 59 \\
\hline \multirow[t]{2}{*}{ 5. WAI versión Observador - Vínculo } & & & & & 1.00 & $.71 * * *$ & $.63 * * *$ & $.84 * * *$ \\
\hline & & & & & & 60 & 60 & 60 \\
\hline \multirow[t]{2}{*}{ 6. WAI versión Observador - Tareas } & & & & & & 1.00 & $.92 * * *$ & $.96^{* * *}$ \\
\hline & & & & & & & 60 & 60 \\
\hline \multirow[t]{2}{*}{ 7. WAI versión Observador - Metas } & & & & & & & 1.00 & $.94 * * *$ \\
\hline & & & & & & & & 60 \\
\hline $\begin{array}{l}\text { 8. WAI versión Observador - Escala } \\
\text { Total }\end{array}$ & & & & & & & & 1.00 \\
\hline
\end{tabular}

Nota. Correlaciones parciales en que se controla el número de sesión en que son aplicados los instrumentos. 56 gl. en cada correlación estimada. *** $\mathrm{p} \leq .001 ; * * \mathrm{p} \leq .01 ; * \mathrm{p} \leq .05 ;{ }^{\mathrm{t}} \mathrm{p} \leq 10$ 
estructura tri-factorial del instrumento (Hatcher y Barends, 1996; Andrusyna et al., 2001).

Dentro del constructo Alianza Terapéutica, al analizar según el método de recolección de datos, se pudo apreciar que las versiones de autoreporte (WAI paciente y terapeuta) presentaron una correlación de magnitud grande, correlación que es superior a aquella obtenida al considerar mediciones que combinen métodos de recolección (observacional y autoreporte), y a la relación obtenida entre ambos métodos observacionales, que alcanzó solo una magnitud moderada. Este patrón de correlaciones podría ser interpretado como que la vivencia en primera persona del proceso terapéutico no puede ser completamente captada por un observador, al acceder a la grabación de la interacción desarrollada por parte del paciente y su terapeuta.

De hecho, los jueces señalaron que les resultó más fácil responder preguntas relacionadas con la sub-escala vínculo, que las de metas y tareas. Esto se podría explicar debido a que el vínculo es algo observable por un tercero en la relación terapéutica, en cambio, si no se habla explícitamente de las tareas y/o metas en la sesión, el puntaje de este aspecto debe ser inferido y/o "completado" por el observador con la información disponible. Por otra parte, se podría postular que pacientes y terapeutas responden este instrumento en forma más impresionista, cargada de las sensaciones y vivencias globales de lo que está ocurriendo en su relación con el otro. A diferencia de lo realizado por los observadores, que responden colocando una atención más racional que busca distinguir entre las dimensiones de la alianza.

Asimismo, las mediciones observacionales de alianza no presentaron una relación con los resultados terapéuticos, a diferencia de las versiones completadas por pacientes y terapeutas; siendo la única excepción la correlación que se presentó entre el vínculo percibido por el observador y el rol social reportado por el paciente. Los resultados obtenidos son coherentes con la literatura que indica que la relación entre alianza terapéutica y resultados depende de la manera en que estos últimos son medidos (Horvarth y Symonds, 1991; Fenton et al., 2001). Así, cuando la alianza y los resultados terapéuticos son auto-reportados por pacientes y terapeutas, la relación es más clara (o de mayor magnitud) que aquella encontrada al utilizar mediciones observacionales de la alianza. Adicionalmente, Fenton el al. (2001) señala que cuando las mediciones de resultados son obtenidas a través de mediciones más objetivas, la alianza medida por los observadores se relacionaría con los resultados terapéutico. Los autores indican que la elevada relación entre alianza terapéutica y resultados evaluados por terapeutas y pacientes podría explicarse por un "Efecto de Halo". Es decir, que la interpretación de la interacción terapéutica está influida por su visión de los resultados de esa interacción cuando son el paciente y el terapeuta los que evalúan.

Dentro de las limitaciones del presente estudio se encuentra el tipo de muestreo (por conveniencia). Sin embargo, debe mencionarse que se habrían presentado problemas de factibilidad al considerar estudios con muestras probabilísticas. De hecho, los procedimientos que requerían que paciente y terapeuta completaran cuestionarios antes y después de la sesión, y además consintieran que se les videograbara dificultaron la recolección de la muestra. Otra limitación del estudio es la desigual distribución de las sesiones consideradas de cada terapia incluida en el estudio. Dos de los procesos terapéuticos aportaron el $30 \%$ de la muestra de sesiones, por lo que los resultados podrían estar particularmente influenciados por lo acontecido en estas terapias. Pese a estas dificultades, la muestra cubre una variedad de motivos de consulta, y fue obtenida en diferentes momentos de la terapia, cubriendo tanto las etapas iniciales, medias como finales en los procesos terapéuticos.

Contar con un instrumento que permite medir alianza terapéutica desde la perspectiva del observador adaptada a la realidad chilena, y que complementa las mediciones de paciente y terapeuta (Santibañez, 2001), permitirá continuar con la investigación en el ámbito de la alianza terapéutica a nivel local.

\section{Referencias}

Alexander, L. B., y Luborsky, L. (1986). The Penn Helping Alliance Scales. En L. S. Greenberg y W. M. Pinsof (Eds.), The psychotherapeutic process: A research handbook (pp. 325-366). New York: Guilford.

Andrusyna, T. P., Tang, T. Z., DeRubeis, R. J., y Luborsky, L. (2001). The factor structure of the Working Alliance Inventory in cognitivebehavioral therapy. Journal of Psychotherapy Practice and Research, $10,173-178$.

Barber, J., Luborsky, L., Crits-Christoph, P., Thase, M., Weiss, R., Frank, A., Onken, L. S., y Gallop, R. (1999). Therapeutic alliance as a predictor of outcome in treatment of cocaine dependence, Psychotherapy Research, 9, 54-73.

Bordin, E. S. (1979). The generalizability of the psychoanalytic concept of the working alliance. Psychotherapy Theory, Research and Practice, $16,252-260$.

Castonguay, L. G., Goldfried, M. R., Wiser, S., Raue, P. J., y Hayes, A. H. (1996). Predicting the effect of cognitive therapy for depression: a study of unique and common factors. Journal of Consulting y Clinical Psychology, 64, 497-504.

Conners, G. J., Carroll. K. M., DiClemente, C. C., Longabaugh, R., y Donovan, D. M. (1997). The therapeutic Alliance and its relationship to alcoholism treatment participation and outcome. Journal of Consulting and Clinical Psychology, 65, 588-598.

Corbella, S., y Botella, L. (2003). La alianza terapéutica: historia, investigación y evaluación. Anales de Psicología, 19, 205-221.

Fenton, L., Cecero, J., Nich, C., Frankforter, T. L., y Carroll, K. M. (2001). Perspective is everything: The predictive validity of six working alliance 
instruments. Journal of Psychotherapy, Practice and Research, 10, 262-268.

Hanson, W., Curry, K., y Bandalos, D. (2002). Reliability generalization of working alliance inventory scale scores. Educational and Psychological Measurement, 62, 659-673.

Hartley, D., y Strupp, H. H. (1983). The therapeutic alliance: Its relationship to outcome in brief psychotherapy. En J. Masling (Ed.), Empirical studies of psychoanalytic theories, 1, 1-37.

Hatcher, R. L., y Barends, A.W. (1996). Patients' view of the alliance in psychotherapy: Exploratory factor analysis of three alliance measures. Journal of Consulting and Clinical Psychology, 64, 1326-1336.

Hatcher, R., y Gillaspy, A. (2006). Development and validation of a revised short version of the Working Alliance Inventory. Psychotherapy Research, 16, 12-55.

Hellerstein, D. J., Rosenthal, R. N., Pinsker, H., Samstag, L. W., Muran, J. C., y Winston, A. (1998). A randomizaed prospective study comparing supportive and dynamic therapies: outcome and alliance. Journal of Psychotherapy, Practices y Research, 7, 261-271.

Horvath, A. O., Del Re, A. C., Flückiger, C., y Symonds, D. (2011). Alliance in Individual Psychotherapy. En J. C. Norcross (Ed.), Psychotherapy relationships that work: Evidence-based responsiveness $\left(2^{\text {nd }}\right.$ ed., pp. 25-69). New York: Oxford University Press.

Horvath, A. O., y Greenberg, L. (1986). The development of the Working Alliance Inventory. En L. S. Greenberg y W. M. Pisnsof, (Eds.), The Psychotherapeutic Process: A Research Handbook (pp. 529-556). Nueva York: Guilford.
Horvath, A. O., y Symonds, B. (1991). Relation between working alliance and outcome in psychotherapy: A metaanalysis. Journal of Counseling Psychology, 39, 32-38.

Horvath, A. O. (1994). Empirical validation of Bordin's pantheoretical model of the alliance: The Working Alliance Inventory perspective. En A. O. Horvath y L. S. Greenberg (Eds), The Working Alliance: Theory, Research, and Practice (pp.109-128). New York: John Wiley y Sons.

Lambert, M. J., y Barley, D. E. (2002). Research summary on the therapeutic relationship and psychotherapy outcome. En J. C. Norcross (Ed.), Psychotherapy relationships that work: Therapist contributions and responsiveness to patients (pp. 17-32). New York: Oxford University Press.

Santibañez, P. (2001). La alianza terapéutica en psicoterapia. Traducción, adaptación y examen inicial de las propiedades psicométricas del "Working Alliance Inventory" en Chile. (Tesis para optar al grado de Magíster), Universidad Católica de Chile, Santiago.

Shelef, K., y Diamond, G. M. (2008). Short form of the revised Vanderbilt Therapeutic Alliance Scale: Development, reliability, and validity. Psychotherapy Research, 18, 433-443.

Tichenor, V., y Hill, C. E. (1989). A comparison of six measures of working alliance. Psychotherapy, 26, 195-199.

Von Bergen, A., y de la Parra, G. (2002). OQ-45.2, cuestionario para evaluación de resultados y evolución en psicoterapia: adaptación, validación e indicaciones para su aplicación e interpretación. Terapia Psicológica, 20, 161-176. 
